\title{
Perceived Air Quality of different sources of smell evaluated by primary school children
}

\author{
Tatiana Armijos Moya ${ }^{1, *}$, Dadi Zhang $^{1}$ and Philomena M. Bluyssen ${ }^{1}$ \\ ${ }^{1}$ Faculty of Architecture and the Built environment, Delft University of Technology, Delft, The Netherlands
}

\begin{abstract}
Our sensory system (nose) could predict the perceived odour intensity of various materials relatively well and in many cases the nose seems to be a better receptor of pollutants than some equipment. To test this ability with children, odour tests were performed as part of a study performed with 335 primary school children in the air quality test chamber of the SenseLab. Two similar experiments were conducted to assess the identification of potentially recognizable odours for children. The different sources of odour were placed in different containers and the children were asked how they feel about the smell and to identify the source of smell. There were statistically significant differences among children's evaluations of different smells and it was difficult for them to identify certain sources of smell.
\end{abstract}

\section{Introduction}

In a recent field study of 54 classrooms of 21 primary schools in the Netherlands, was found that among the 1145 children studied (average 10 years old), $67 \%$ of the children was bothered by smells (girls $71 \%$ and boys $62 \%)$. The most frequently occurring smells in the classroom according to the children were 'human' (56\%) and 'stuffy' $(27 \%)[1]$.

To investigate preferences and needs, but also responses to single components and interactions of different environmental conditions more in depth, 335 children from the previous studied schools, were invited to take part in a series of tests in a semi-laboratory environment: the SenseLab. The SenseLab comprises of four test chambers (one for each IEQ factor: air, light, acoustics and thermal aspects) and the Experience room (a room for integral perception) [2]. In the air quality chamber different sources of smell were tested. This paper describes the early results of this study.

\section{Methodology}

This study was part of a series of tests performed with children from the previous studied schools, in the SenseLab [2]. During the winter and spring of 2018, 335 students of 21 schools in the Netherlands visited the SenseLab to execute some experiments and questionnaires on the health and comfort of school children of group 7 and 8 (8-13 years old). The selected schools for these experiments were used in previous tests and the recruitment of these schools was on voluntary basis [1].

Previous studies have demonstrated the ability to identify odours increases with age in children. This is due to an ongoing process of odour learning rather than an actual increase in olfactory function [3]. The aim during the development of these tests for children was to include only odours that are well known and able to be correctly identified by a majority of children.

Two similar experiments were conducted to assess the identification of potentially recognizable odours for children. Every session, five odorant elements were used. For the air quality test chamber (Figure1), different olfactory stimuli were selected to be identified for the children (Table 1). These odorants were selected based on previous studies in Dutch schools [1]. Every time three to four children entered the chamber and they were asked to answer a questionnaire regarding the smell of different elements/materials in combination of interactive techniques to recognize the smell.

The stimuli were placed in four different covered plastic containers located in the "sniffing table" with sniffing cones [5], above which the children could take a sniff when activated (Figure 2). At the same time, one of the materials located in the plastic containers was also located in the CLIMPAQ $50 \mathrm{~L}$ (Figure 1), which is a small test chamber that allows to analyse emissions and pollutants from a wide range of materials. The children were asked to match the odour with one of the plastic containers.

The children were asked: "How do you like the smell?" and "Can you tell what it is?". When working with children, who are not always able to clearly communicate and express how they feel, the use of graphic questionnaires could be an alternative option to obtain more information about their experiences. The perceived odour was assessed on a five-graphic-grade scale. Before administering the questionnaire in the air quality chamber, it was distributed and tested among the staff, in order to improve and adapt it with the

\footnotetext{
* Corresponding author: T.E.ArmijosMoya@tudelft.nl
} 
Table 1. Selected olfactory stimuli.

\begin{tabular}{|c|c|c|}
\hline Container No. & Material & Notes \\
\hline 1 & Perfume & New leaves every session \\
\hline 2 & Mint leaves & Varied according to schedule \\
\hline 3 & Material: carpet/MDF/vinyl & \\
\hline 4 & Crayons & Varied according to schedule \\
\hline
\end{tabular}

comments. During the survey, before the questionnaire was distributed, an explanation was given of the contents and purpose of the questionnaire, after which the questionnaires were distributed. In general, it took the children five minutes to perform the test and fill in the questionnaire.

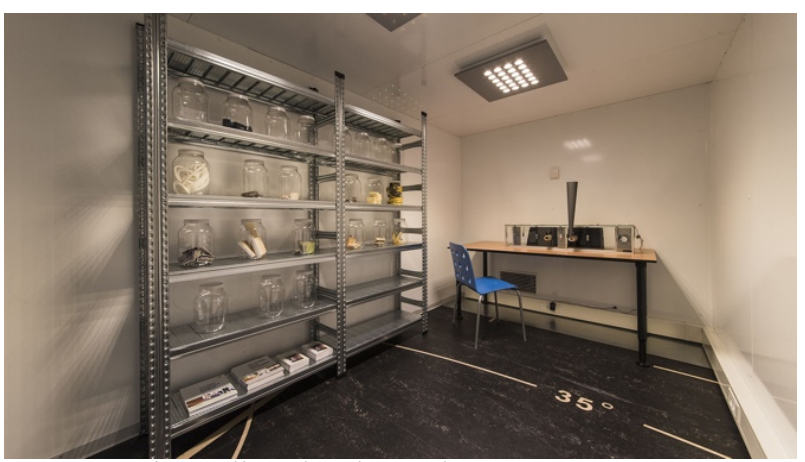

Figure 1. Air Quality Chamber and CLIMPAQ 50L [4].

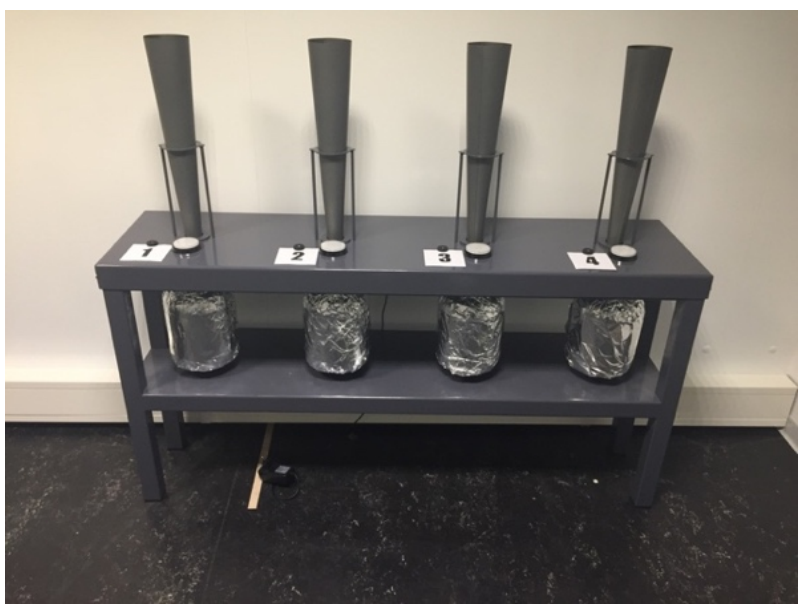

Figure 2. Sniffing Table.

\section{Results}

During these tests, children executed smell identification tests, where four smell stimuli were placed inside four different containers.

First results of the ANOVA test performed, showed that there were statistically significant differences among children's evaluations of different smells. They liked the source of smell in container 1 (perfume) most, and then mint leaves, and then material/carpet/vinyl, and they liked crayons the least.

More details of the results will be presented on the poster and in a journal paper [6].

\section{Conclusions}

The study presented was part of a larger study in which primary school children performed several tests in four

test chambers and a workshop in the experience room. This study was a first attempt to study the sensory evaluation of children and apply these methods in protocols of future experiments.

\section{References}

1. P.M. Bluyssen, D. Zhang, S. Kurvers, M. Overtoom, M. Ortiz-Sanchez, Self-reported health and comfort of school children in 54 classrooms of 21 Dutch school buildings, Building and Environment 138:106-23 (2018).

2. P.M. Bluyssen, F. van Zeist, S. Kurvers, M. Tenpierik, S. Pont, B. Wolters, L. van Hulst, D. Meertins, The creation of SenseLab: a laboratory for testing and experiencing single and combinations of indoor environmental conditions, Intelligent Building International 10(1):5-18 (2018).

3. V.A. Schriever, E. Agosin, A. Altundag, H. Avni, H.C. Van, C. Cornejo, G. de los Santos, G. Fishman, C. Fragola, M. Guarneros, N. Gupta, R. Hudson, R. Kamel, A. Knaapila, I. Konstantinidis, B.N. Landis, M. Larsson, J.N. Lundstrom, A. Macchi, F. MarinoSanchez, L. Martinec Novakova, E. Mori, J. Mullol, M. Nord, V. Parma, C. Philpott, E.J. Propst, A. Rawan, M. Sandell, A. Sorokowska, P. Sorokowski, L.M. Sparing-Paschke, C. Stetzler, C. Valder, J. Vodicka, T. Hummel, Development of an International Odor Identification Test for Children: The Universal Sniff Test. J Pediatr-Us 198:265-272 (2018)

4. TUDelft, Test Chambers TU Delft2018, Available from:

https://www.tudelft.nl/bk/onderzoek/onderzoeksfacil iteiten/senselab/test-chambers/.

5. P.M. Bluyssen, Air quality evaluated by a trained panel (Doctoral Dissertation), Lyngby, Technical University of Denmark. (1990).

6. T.E. Armijos Mayo and P.M. Bluyssen, Appraisal of different sources of smell by primary school children in the SenseLab (submitted).

\footnotetext{
* Corresponding author: T.E.ArmijosMoya@tudelft.nl
} 